\title{
Modeling Flow Rate to Estimate Hydraulic Conductivity in a Parabolic Ceramic Water Filter
}

lleana Wald

University of South Florida

\author{
Advisors: \\ Brian Curtin, Mathematics and Statistics \\ Scott Campbell, Chemical \& Biomedical Engineering \\ Problem Suggested By: Scott Campbell
}

Follow this and additional works at: https://digitalcommons.usf.edu/ujmm

Part of the Mathematics Commons

UJMM is an open access journal, free to authors and readers, and relies on your support:

Donate Now

\section{Recommended Citation}

Wald, Ileana (2012) "Modeling Flow Rate to Estimate Hydraulic Conductivity in a Parabolic Ceramic Water Filter," Undergraduate Journal of Mathematical Modeling: One + Two: Vol. 4: Iss. 2, Article 6.

DOI: http://dx.doi.org/10.5038/2326-3652.4.2.6

Available at: https://digitalcommons.usf.edu/ujmm/vol4/iss2/6 


\title{
Modeling Flow Rate to Estimate Hydraulic Conductivity in a Parabolic Ceramic Water Filter
}

\author{
Abstract \\ In this project we model volumetric flow rate through a parabolic ceramic water filter (CWF) to determine \\ how quickly it can process water while still improving its quality. The volumetric flow rate is dependent \\ upon the pore size of the filter, the surface area, and the height of water in the filter (hydraulic head). We \\ derive differential equations governing this flow from the conservation of mass principle and Darcy's Law \\ and find the flow rate with respect to time. We then use methods of calculus to find optimal specifications \\ for the filter. This work is related to the research conducted in Dr. James R. Mihelcic's Civil and \\ Environmental Engineering Lab at USF.
}

\section{Keywords}

Flow Rate, Hydraulic Conductivity, Water Filter

\section{Creative Commons License} (c) $($ i) $(9)$

This work is licensed under a Creative Commons Attribution-Noncommercial-Share Alike 4.0 License. 


\section{TABLE OF CONTENTS}

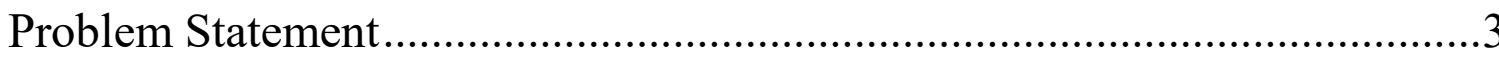

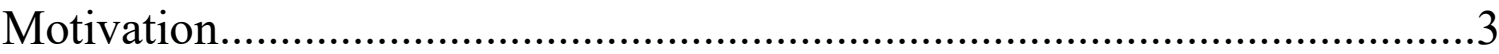

Mathematical Description and Solution Approach .......................................5

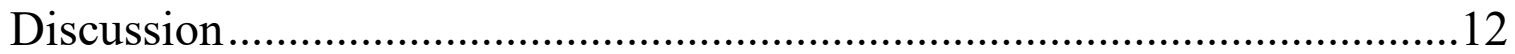

Conclusion and Recommendations .........................................................13

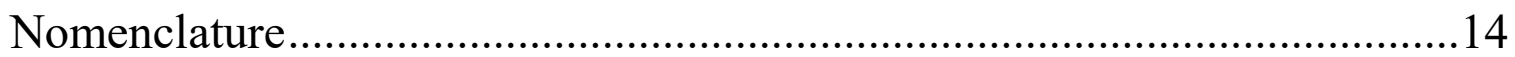

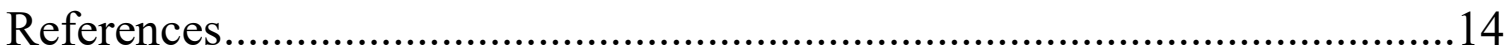




\section{PROBLEM STATEMENT}

Optimal flow rate is an important quality control standard for manufacturers of point-ofuse ceramic water filters. Can volumetric flow rate be modeled as a function of time and height of water in the filter? Using integral and differential calculus, a differential equation can be derived that approximates flow rate of water through the paraboloid-shaped filter, yielding a hydraulic conductivity variable $\alpha$.

\section{MOTIVATION}

According to the World Health Organization and UNICEF's Joint Monitoring Programme, 884 million people do not have access to improved sources of drinking water (WHO/UNICEF). Lack of access to safe drinking water is one of the main causes of disease in developing countries. Household water treatment technologies, termed point-of-use (POU) technologies, have been developed in an effort to solve this problem and improve people's access to clean and safe drinking water. An important branch of research in this area involves testing the hydraulic performance of point of use ceramic water filters (CWFs) designed for use in developing communities.

The technology of ceramic water filters is simple: clay is mixed with an organic (burnout) material, such as cornhusks or saw dust, which will burn out during the firing and leave small pores throughout the filter. All of the production materials are usually locally and sustainably sourced. The filter is molded into a bowl (paraboloid) shape and then fired to a temperature sufficiently high to completely vitrify the ceramic elements and burn out all organic matter. Once fired, the filter should remain slightly porous so that water may filter at an 
acceptable flow rate. This flow rate is used as a

production standard for many manufacturers of POU ceramic filters, including Potters for Peace and FilterPure. These ceramic filters treat water through physical filtration and also disinfect the water through the use of impregnated or coated colloidal silver. The small pores in the filter "strain" out dirt and bacteria while the colloidal silver acts as a chemical biocide to kill microbes. Ceramic water filters have proven to be tremendously effective in reducing the exposure of users to contaminated water and the incidence

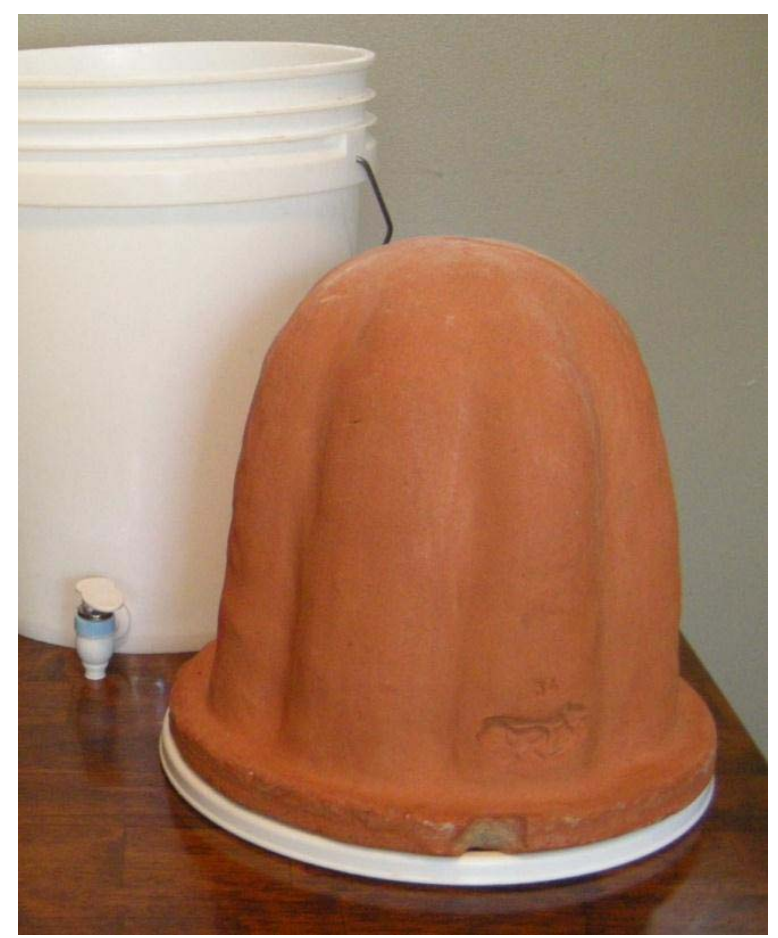

Figure 1: FilterPure filter sitting bottom up on a bucket lid. of diarrhea over an extended period of time (Brown and Sobsey; Hagan, Harley and Pointing).

In order to ensure that the filters are effective and safe to use in the household, manufacturers must have a rigorous quality control process. One important quality control measure is the first hour flow rate test. Manufacturers aim for an optimal flow rate of water out of the filter of 1 to 2.5 liters per hour. Flow rate is an important aspect of the functionality of the filter. If the flow rate is too high, there is likely a crack or the pore size is too large and will not be effective in filtering out of dirt and bacteria. If the flow rate is too low, then the filters will be inconvenient for household users to use on a daily basis and will likely not be adopted.

First hour flow rate can also serve as an important comparative tool to assess the hydraulic conductivity of filters and compare with others in order to improve their performance. By adapting Darcy's Law to a paraboloid geometry and relating it to the FilterPure filters' 
experimental volumetric flow rate, a hydraulic conductivity variable $\alpha$ can be determined. The volumetric flow rate is dependent upon the pore size of the filter, amount of surface area, and the hydraulic head of the water in the filter. Using the theory of conservation of mass, which states that the rate of mass into the vessel minus the rate of mass out of the vessel equals the rate of accumulation of mass, equations can be derived using differential and integral calculus to determine the flow rate of water through a vessel as a function of time.

\section{MATHEMATICAL DESCRIPTION AND SOLUTION APPROACH}

The law of conservation of mass, also known as the principle of mass/matter conservation, states that the mass of an isolated system (closed to all matter and energy) will remain constant over time, yielding the following:

$$
\text { (rate of mass in })-(\text { rate of mas out })=\text { rate of accumulation of mass. }
$$

In practice, the ceramic water filter is filled up and then left undisturbed to filter water. It is not, presumably, continually filled to the top. Once the filter has been filled up and begins to filter water, we can say that in the relationship described by the law of conservation of mass, the rate of mass in, equals zero, so:

$-($ rate of mas out $)=$ rate of accumulation of mass

This relationship gives us the following equation that states that the density of the fluid $\rho$ multiplied by the volumetric flow rate of fluid out of the vessel $Q$ equals the rate of accumulation of liquid outside the filter with respect to time: 


$$
-\rho Q=\frac{d M}{d t}
$$

We let $\rho=1000 \mathrm{~kg} / \mathrm{m}^{3}$ represent the density of water and set the mass $M$ of liquid accumulation outside filter equal to density $\rho$ times the volume $V$ in $\mathrm{mL}$ of liquid:

$$
-M=\rho V
$$

By integrating the circular cross-sectional areas with respect to height (represented by the variable $z$ ) of a paraboloid we can find its volume at any height:

$$
V(h)=\int_{0}^{h} A d z
$$

where $A=\pi r^{2}$. We can also find the corresponding radius $r$ for any height $z$ using the maximum filter radius $R$ and the maximum filter height $H$. Let

$$
r=k z^{1 / 2}
$$

then at $z=H$ we have $r=R$ so $R=k H^{1 / 2}$. Thus, $k=\frac{R}{H^{1 / 2}}$ and substituting this into Equation 4 we have $r=R\left(\frac{z}{H}\right)^{1 / 2}$. Since the area of a circle is $A=\pi r^{2}$, we can write:

$$
A=\pi\left[R\left(\frac{Z}{H}\right)^{1 / 2}\right]^{2}
$$

Now, substituting Equation 5 into Equation 3, we get:

$$
V(h)=\int_{0}^{h} \pi R^{2} \frac{z}{H} d z=\left.\frac{\pi R^{2}}{H} * \frac{z^{2}}{2}\right|_{0} ^{h}=\frac{\pi R^{2} h^{2}}{2 H}
$$

a simplified equation for the volume. 


\section{Relation of rate of change of mass to rate of change of volume:}

Now that we have derived an equation for the volume of a paraboloid, the rate of change of that volume can be related to the rate of change of mass (Darcy's law).

Recall Equation 1 relating the rate of change of mass to volumetric flow rate: $-\rho Q=\frac{d M}{d t}$.

We can also say that,

$$
\frac{d M}{d t}=\rho \frac{d v}{d t}=\rho \frac{d v}{d h} \frac{d h}{d t}
$$

and substituting the derivative of the volume function (Equation 6) into Equation 7, for $\frac{d v}{d h}$, we get $\frac{d M}{d t}=\rho\left[\frac{2 h \pi R^{2}}{2 H}\right] \frac{d h}{d t}$, which, simplified, is $\frac{d M}{d t}=\rho \frac{h \pi R^{2}}{H} \frac{d h}{d t}$. Now substituting into Equation 1 for $\frac{d M}{d t}$, we obtain

$$
\frac{h \pi R^{2}}{H} \frac{d h}{d t}=-Q
$$

an equation describing flow rate.

\section{Velocity of water flow:}

Darcy's Law is well known and states that the flow speed through a porous medium is directly proportional to the pressure gradient through that medium and to the square of the characteristic size of the pore spaces of the medium, and inversely proportional to the viscosity of the medium. The value of the constant is different for different porous media, because of the differences in particle shape and packing geometry. The constant, c, in the equation below 
depends on the size distribution of the porous medium as well as on the particle shape and packing (Southard):

$$
v=c \Delta P
$$

describing the velocity of water flow out of the wall of a ceramic water filter; here, $c$ is the proportionality constant and $\Delta P$, the change in pressure, is the difference of atmospheric pressure from the total fluid pressure inside the filter, $\Delta P=P-P_{a t m}$.

The total fluid pressure at any point of the water level in the filter is equal to the atmospheric pressure pushing down on fluid from above the surface of water in the filter plus the pressure of water above the point of interest. Recall that pressure exerted by a static fluid is dependent upon the depth of the fluid, the density of the fluid and the acceleration due to gravity. We can write $P=P_{\text {atm }}+\rho g(h-z)$, where $g$ is the acceleration due to gravity, $h$ is height of water in the filter and $z$ is the change in height while water is flowing out of the filter. Subtracting atmospheric pressure from total pressure yields

$$
P-P_{a t m}=\rho g(h-z)
$$

and using Equation 10 with 9 we see that $v=c \rho g(h-z)$. If we combine density, acceleration due to gravity and the proportionality constant into the coefficient of volumetric flow rate $\alpha$, we obtain

$$
v=\alpha(h-z) .
$$




\section{Flow of water through filter walls:}

To calculate the volumetric flow rate $Q$ of water through the walls of the filter, we need the function of flow rate through the walls of a "slice" of the paraboloid. The volumetric flow rate through a slice is equal to the velocity of water flow through the filter wall at the slice integrated with respect to the (surface) area of the slice $(d A)$ :

$$
Q_{\text {slice }}=\int_{A} v d A
$$

and with Equation 11,

$$
Q_{\text {slice }}=\int_{A} \alpha(h-z) d A \text {. }
$$

The corresponding area depends on the arc length along the paraboloid and on the circumference of the corresponding circular cross-sections, so that the area differential is

$$
d A=2 \pi r \sqrt{1+\left(\frac{d r}{d z}\right)^{2}} d z
$$

and substituting Equation 14 into 13, we get

$$
Q=2 \pi \int_{0}^{h} r \sqrt{1+\left(\frac{d r}{d z}\right)^{2}} \alpha(h-z) d z
$$

From the discussion of Equation 4, recall that

$$
r=R\left(\frac{Z}{H}\right)^{1 / 2}=R \frac{z^{1 / 2}}{H^{1 / 2}}
$$

and the derivative of Equation 16 with respect to $z$ is 


$$
\frac{d r}{d z}=\frac{1}{2} R \frac{1}{z^{1 / 2} H^{1 / 2}}
$$

so that $\left(\frac{d r}{d z}\right)^{2}=\frac{R^{2}}{4 z H}$. Then, substituting this into Equation 15, we get

$$
Q=2 \pi \alpha \int_{0}^{h} R \frac{z^{1 / 2}}{H^{1 / 2}} \sqrt{1+\frac{R^{2}}{4 z H}}(h-z) d z
$$

which can be written as

$$
Q=2 \pi \alpha R \int_{0}^{h}(h-z) \sqrt{\frac{z}{H}+\frac{R^{2}}{4 H^{2}}} d z
$$

To integrate Equation 18 with respect to $z$, we introduce the following constants: $a=\frac{1}{H}$

and $b=\frac{R^{2}}{4 H^{2}}$. Then,

$$
\int_{0}^{h}(h-z) \sqrt{a z+b} d z=\left.\frac{2(a z+b)^{3 / 2}(5 a h-3 a z+2 b)}{15 a^{2}}\right|_{0} ^{h}
$$

or, simplified,

$$
Q=\frac{4 \pi \alpha R}{15 a^{2}}\left[(a h+b)^{3 / 2}(2 a h+2 b)-b^{3 / 2}(5 a h+2 b)\right]
$$

and combining (20) and (8) we get

$$
\frac{h \pi R^{2}}{H} \frac{d h}{d t}=-\frac{4 \pi \alpha R}{15 a^{2}}\left[(a h+b)^{3 / 2}(2 a h+2 b)-b^{3 / 2}(5 a h+2 b)\right] .
$$

Rearranging the terms and integrating with $h_{f}=$ height of liquid at some time $t$ : 


$$
-\int_{H}^{h_{f}} \frac{\pi R^{2} h}{H} \times \frac{15 a^{2}}{4 \pi R} \times \frac{1}{\left[(a h+b)^{\frac{3}{2}}(2 a h+2 b)-b^{\frac{3}{2}}(5 a h+2 b)\right]} d h=\int_{0}^{t} \alpha d t
$$

Now integrate the right side of Equation 22 with respect to time:

$$
-\int_{H}^{h_{f}} \frac{\pi R^{2} h}{H} \times \frac{15 a^{2}}{4 \pi R} \times \frac{d h}{\left[(a h+b)^{\frac{3}{2}}(2 a h+2 b)-b^{\frac{3}{2}}(5 a h+2 b)\right]}=\alpha t
$$

so that we can solve for $\alpha$, giving:

$$
\alpha=-\frac{1}{t} \int_{H}^{h_{f}} \frac{\pi R^{2} h}{H} \times \frac{15 a^{2}}{4 \pi R} \times \frac{d h}{\left[(a h+b)^{\frac{3}{2}}(2 a h+2 b)-b^{\frac{3}{2}}(5 a h+2 b)\right]}
$$

equivalently,

$$
\alpha=\frac{1}{t} \int_{h_{f}}^{H} \frac{\pi R^{2} h}{H} \times \frac{15 a^{2}}{4 \pi R} \times \frac{d h}{\left[(a h+b)^{\frac{3}{2}}(2 a h+2 b)-b^{\frac{3}{2}}(5 a h+2 b)\right]} .
$$

Numerical integration to solve for $\alpha$ and calculate the coefficient of volumetric flow rate:

As mentioned previously, according to Darcy's Law, the constant or intrinsic permeability of the porous medium depends on the particular porous medium. For this project we calculate a coefficient that is based on the average experimental flow rate of a FilterPure ceramic water filter and its dimensions.

Recall that $a=\frac{1}{H}$ and $b=\frac{R^{2}}{4 H^{2}}$. Since $R$ and $H$ are both constants, we can substitute the actual filter dimensions and solve for alpha using Microsoft Excel and trapezoidal integration. The Trapezoidal Rule is an approximate method for calculating definite integrals (Stewart). In 
general, the area of the trapezoid that lies about the $i$ th subinterval is $\frac{1}{2} \Delta x\left[f\left(x_{i-1}\right)+f\left(x_{i}\right)\right]$ and the sum of all resulting trapezoids approximates the value of the definite integral.

Let the filter's specifications be as follows: $t=1 \mathrm{hr}, \mathrm{R}=13.2 \mathrm{~cm}, H=25.5 \mathrm{~cm}$. Then $a=\frac{1}{H}=0.039216 \mathrm{~cm}^{-1}$ and $b=\frac{R^{2}}{4 H^{2}}=0.06699$. Using these numbers with Equation 24 and simplifying algebraically (all numbers rounded to 5 significant digits, see Appendix), we obtain the following expression for $\alpha$ :

$$
\alpha=\int_{24.85}^{25.5} \frac{0.00299 d h}{\left[(0.03922 h+0.06699)^{3 / 2}(0.07843 h+0.13398)-0.017339(0.19608 h+0.13398)\right]}
$$

and the trapezoidal approximation gives $\alpha=0.022603 \mathrm{hr}^{-1}$.

\section{DISCUSSION}

The first hour flow rate test is useful, but does have a limitation: it is conducted when the filter has just been filled and does not account for the decrease of head with time and thus the decrease in flow rate. Still, if we can calculate the coefficient of hydraulic conductivity using this test, we have a valuable tool for comparing flow rates through different types of filters and ceramic mediums.

It should be noted that the thickness of the ceramic walls was not accounted for in the equations. Wall thickness tends to vary throughout the filter (usually 1-3 cm), but an average thickness could be calculated and included in the model to obtain a more accurate estimation for the coefficient of hydraulic conductivity. Porosity of the medium was also not accounted for in this model and should definitely be considered as a parameter in future studies. 


\section{CONCLUSION AND RECOMMENDATIONS}

Despite these limitations, this model can still serve as a tool to manufacturers and researchers working to improve the performance of CWFs. The average first hour flow rate for this model was $354 \mathrm{~mL}$ per hour (determined by using the known constant values and the heights listed in the Appendix with Equation 20) - well below the minimum target flow rate of $1000 \mathrm{~mL}$ per hour. If CWFs consistently filter water at slow rates, the user acceptability of this technology in developing communities may be severely impacted. Future studies could benefit from developing models similar to this one and testing various shapes and ceramic and burnout materials to continue to optimize flow rate and microbial effectiveness. 


\section{NOMENCLATURE}

\begin{tabular}{|c|c|c|}
\hline Symbol & Term & Units \\
\hline$M$ & Mass of liquid & $\mathrm{kg}$ \\
\hline$V$ & Volume & $\mathrm{cm}$ \\
\hline$Q$ & Volumetric flow rate of liquid & $\mathrm{cm}^{3} / \mathrm{hr}$ \\
\hline$R$ & Maximum radius of filter & $\mathrm{cm}$ \\
\hline$H$ & Maximum height of filter & $\mathrm{cm}$ \\
\hline$h$ & Height of liquid in filter & $\mathrm{cm}$ \\
\hline$Z$ & Change in height of fluid in filter & $\mathrm{cm}$ \\
\hline$t$ & Time & $\mathrm{hr}$ \\
\hline$P$ & Pressure & $\mathrm{Pa}$ \\
\hline$v$ & Velocity & $\mathrm{m} / \mathrm{s}$ \\
\hline$\rho$ & Density of water & $\mathrm{kg} / \mathrm{m}^{3}$ \\
\hline$\alpha$ & Coefficient of volumetric flow & $\mathrm{hr}$ \\
\hline$a$ & Constant & $\mathrm{cm}^{-1}$ \\
\hline$b$ & Constant & $\mathrm{n} / \mathrm{a}$ \\
\hline
\end{tabular}

\section{REFERENCES}

Brown, Joe and Mark Sobsey. "Independent Appraisal of Ceramic Water Filtration Interventions in Cambodia: Final Report." UNICEF - Cambodia (2006).

Hagan, Judy, et al. "Ceramic Water Filter Handbook." 2009. Engineering for Change. Resource

Development International - Cambodia, Engineers Without Borders Australia. December 2012

$<$ https://www.engineeringforchange.org/static/content/Water/S00067/Ceramic\%20filter\%20manu al\%20no-appendices.pdf $>$.

Southard, John. "Special Topics in Earth, Atmospheric, and Planetary Sciences: The Environment of the Earth's Surface." 2007. MIT Open Courseware. December 2012

$<$ http://ocw.mit.edu/courses/earth-atmospheric-and-planetary-sciences/12-090-special-topics-inearth-atmospheric-and-planetary-sciences-the-environment-of-the-earths-surface-spring2007/lecture-notes/earthsurface 4.pdf>.

Stewart, James. Essential Calculus: Early Transcendentals. 5th. Brooks Cole, Cengage Learning, 2006.

WHO/UNICEF. "Progress on sanitation and drinking-water 2010 update." 2010. World Health Organization. Joint Monitoring Programme for Water Supply and Sanitation, Geneva, Switzerland. December 2012

$<\underline{\text { http://www.who.int/water_sanitation_health/publications/9789241563956/en/index.html>. }}$. 